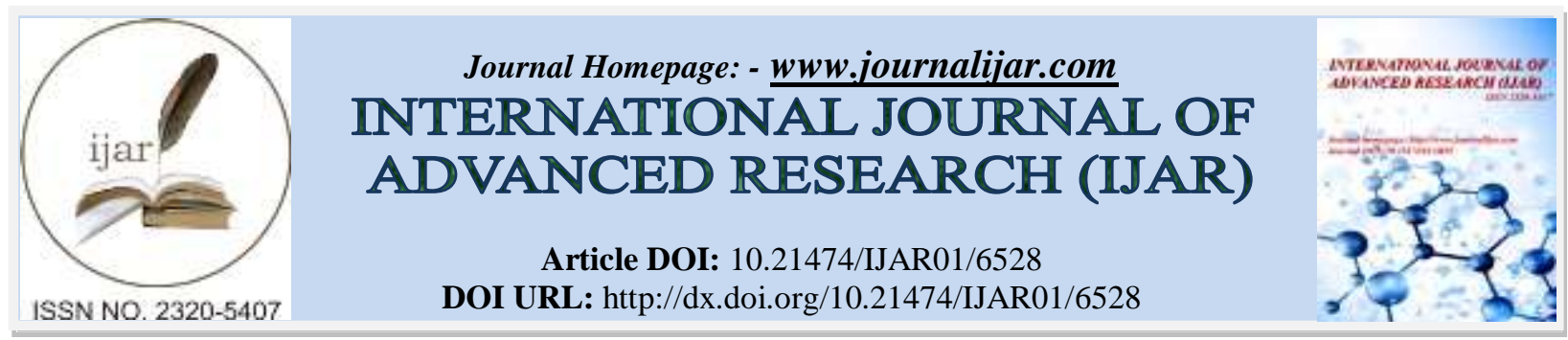

RESEARCH ARTICLE

\title{
ANTERIOR FIXED FUNCTIONAL SPACE MAINTAINER; NOVEL AESTHETIC APPROACH: A CASE
} SERIES.

\section{Dr. Jimisha Patel ${ }^{1}$, Dr. Mayur Vaghasiya ${ }^{1}$, Dr. Pooja Patel ${ }^{1}$, Dr. Shoba Fernandes ${ }^{2}$ and Dr. Jayasudha Kalgudi ${ }^{3}$.}

1. Post Graduate Student, Department of Pediatric and Preventive Dentistry, Narsinhbhai Patel Dental College and Hospital, Visnagar, India.

2. Professor and Head, Department of Pediatric and Preventive Dentistry, Narsinhbhai Patel Dental College and Hospital, Visnagar, India.

3. Professor, Department of Pediatric and Preventive Dentistry, Narsinhbhai Patel Dental College and Hospital, Visnagar, India.

\section{Manuscript Info}

Manuscript History

Received: 14 December 2017

Final Accepted: 16 January 2018

Published: February 2018

Key words:-

Primary anterior teeth trauma, Space maintainer, Esthetic.

\begin{abstract}
Traumatic dental injury is one of the main causes of premature loss of maxillary anterior teeth in school going children, causing psychological trauma both to patient as well as parents. In that situation, choice of treatment is maintaining the space by fixed/removable or functional/nonfunctional appliances ${ }^{1}$. A variety of therapeutic modalities, from removable partial dentures to conventional fixed space maintainer can be used for the replacement of traumatically missing or carious lost primary anterior teeth ${ }^{2}$. In anterior region, esthetics is an important concern along with function and space management. Fiberreinforced composite (FRC) space maintainer solves all these purposes efficiently and effectively. In addition, the technique is simple and the appliance is very comfortable inside the oral cavity ${ }^{3}$. This case series of premature loss of anterior primary teeth which were replaced by FRC retained esthetic functional space maintainers. The appliances were found to be functioning satisfactorily upon follow-up of 1 Year.
\end{abstract}

Copy Right, IJAR, 2018,. All rights reserved.

\section{Introduction:-}

Traumatic injuries to primary teeth are common, especially 18 -30 months children are very prone to accidents, and in particular maxillary anterior teeth are more susceptible for injuries, where avulsion accounts for primary teeth is around $9-12 \%$. Early loss of maxillary incisors can cause psychological, aesthetic and functional problem due to development of tongue thrusting habit in edentulous space ${ }^{4}$.

Tooth material arch length discrepancy is the most common causes for malocclusion. An excess arch length or space as compared to tooth material results in spacing in the dentition. Premature loss of primary tooth is one of the etiologies for the loss of space or arch length. When a primary tooth is lost prematurely the teeth adjacent to the created space tend to drift into the space resulting in the loss of space required for the proper alignment of the succedaneous teeth in the arch, thereby resulting in crowding and other types of malocclusion. In the situation where an anterior primary tooth is lost before schedule, the drifting of adjacent teeth into the space rarely occurs, thus

Corresponding Author:-Dr. Jimisha Patel.

Address:- Post Graduate Student, Department of Pediatric and Preventive Dentistry, Narsinhbhai Patel 1051 
making space management a minimal concern to the pedodontist. In contrast, the premature loss of an anterior tooth definitely results in an unesthetic smile and difficulty in biting, i.e. loss of function thus making the situation which cannot be left unattended ${ }^{3}$.

The primary anteriors are lost most commonly at the age of 2 to 4 years, because of trauma or caries. This is the age, when the child starts moving out of his house and he or she starts indulging in various outdoor and sports activities. Loss of an anterior tooth at this age, apart from the functional problems associated with it, may result in psychological trauma to the child ${ }^{3}$.

A variety of appliances have been developed by various investigators to covenant with space management in case of early loss of a primary tooth. The choice can be between a removable and a fixed one; or it can be unilateral or bilateral; and functional or nonfunctional. The selection of the appliance depends upon a number of factors including the child's stage of dental development, the dental arch involved, the tooth missing and the status of the teeth adjacent to the lost tooth ${ }^{5}$.

Over the past few years, the development of Fiber-Reinforced Composites (FRC) has become accessible to the dental profession with the possibility of fabricating adhesive, esthetic, and metal-free tooth replacements even in the case of molar teeth ${ }^{3}$.

The FRC is made up of two components: the fibers and the resin matrix. The resin matrix serves as a carrier, protector, and load-splicing medium around the fibers. To improve the mechanical properties of composite resins and to optimize the mechanical behavior of the material, specifically-oriented filler materials, such as glass fibers, aramid fibers, carbon/graphite fibers, and ultra high molecular weight polyethylene (UHMWPE) fibers, have been anticipated ${ }^{6}$.

In the present case series report of three cases with simple modification of fixed space maintainers is discussed.

\section{Case Report 1:-}

A 5-year-old boy reported to the dental clinic with history of pain in the upper anterior tooth since 2 days during mastication. The parents reported that he had a fall while playing at home before 8 month ago. The tooth was mildly symptomatic for few days but symptoms subsided thereafter. Parents did not report to any dentists at the time of trauma.

On clinical examination, the maxillary left primary central incisor(61) was mobile (grade III) and was tender on palpatio. On Radiographic examination showed considerable bone loss around the 61. Extraction of the tooth followed by a fixed esthetic functional space maintainer was decided as the treatment plan for the region. The procedure was carried out under local anesthesia and the tooth was extracted atraumatically. The patient was kept on weekly recall.

After 2 weeks, it was observed that socket healed uneventfully(Figure 1). A strip of FRC resin was cut approximately of the length equal to the distance from the distal surface of the right upper central incisor to the distal surface of the left upper lateral incisor. The palatal surfaces of 51 and 62 were conditioned with 37\% phosphoric acid. Bonding agent was applied and was cured. A thin layer of flowable Composite resin was applied over the etched surfaces of the abutment teeth. Following this the FRC strip was adapted over the palatal surface extending from the distal surface of the 51 through the distal surface of the 62. Finally, strip was placed in position cured with visible light.

The tooth was prepared with composite resin. For this, cellulose strip was utilized as a form and composite resin of appropriate shade was cured in small increments. Then finishing and polishing of the tooth was done to achieve morphological similarity to the natural tooth(Figure 2). The occlusion was checked to correct any pre-mature contact. 


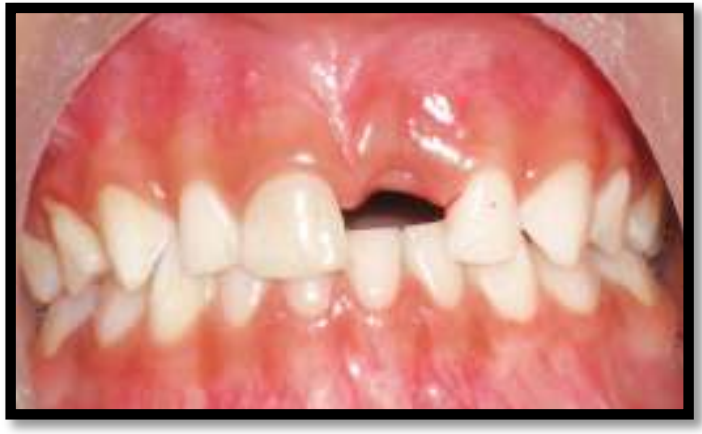

Fig. 1:- Preoperative photograph of Healed Socket

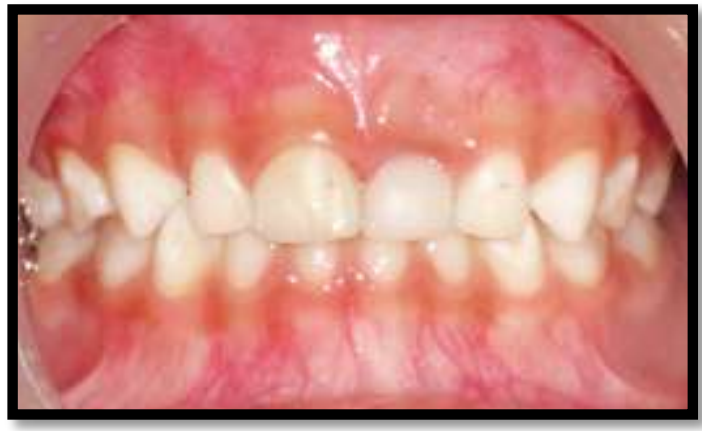

Fig. 2:- Fixed Functional appliance

\section{Case Report 2:-}

A 4 year old male patient reported to department of Pediatric and Preventive dentistry, NPDCH, Visnagar with the chief complaint of broken teeth in relation to upper front tooth region since last 2 to 3 months. Patient gave history of trauma before 1 and half years and patient did not consult any dentist at that time. There was no history of any associated symptoms. Over the past two weeks, patient developed history of pain in this same region, hence Patient reported to dental clinic.

On clinical examination, presence of grossly carious upper 61 with grade 1 mobility was observed. Radiographic examination showed bone loss around 61. Extraction of teeth followed by a fixed esthetic functional space maintainer was decided as the treatment plan for the tooth. The extraction was carried out under local anesthesia The patient was kept on weekly recall.

After 2 weeks, uneventful healing of socket was observed. Impression was made by rubber base impression material in order to prepare the appliance in indirect method. Appropriate shade was selected and tooth fabricated. The fiber reinforced composite resin splint was cut according to required length. This splint was bonded to prepared tooth and light cured. Finishing and polishing was done(Figure 1\&2). The prepared appliance was then bonded to the adjacent teeth and cured intra orally(Figure 3). Occlusion was checked for any premature contact.Patient was recalled after 1 week for appliance follow up. The appliance was esthetically and functionally stable effectively. Patient was kept on 3 month follow up period.

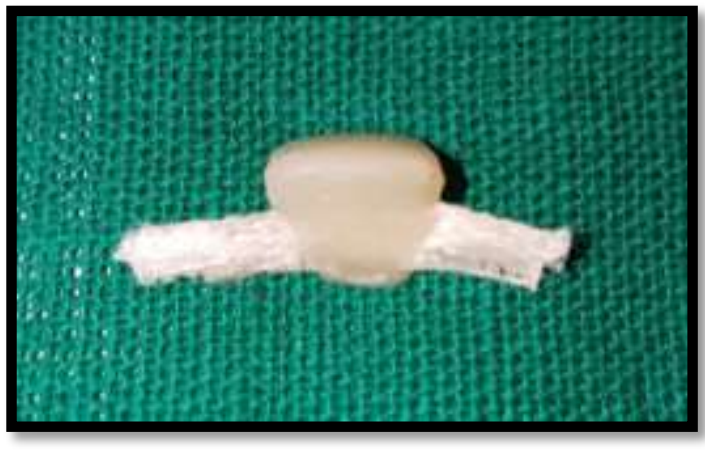

Fig 1:- Prepared Fixed functional appliance

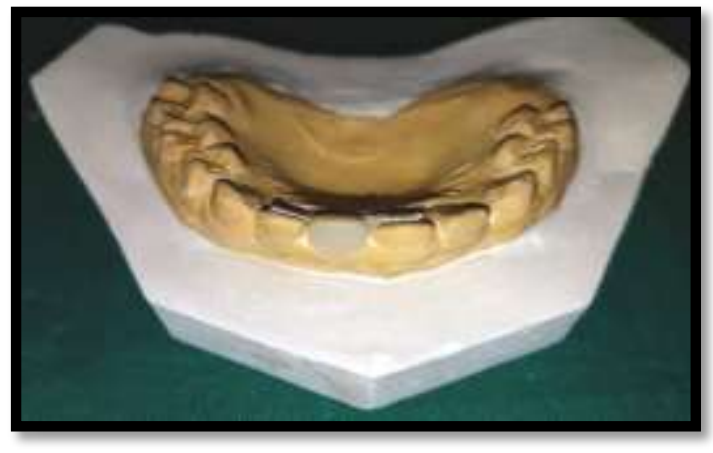

Fig 2:- Fixed functional appliance on cast 


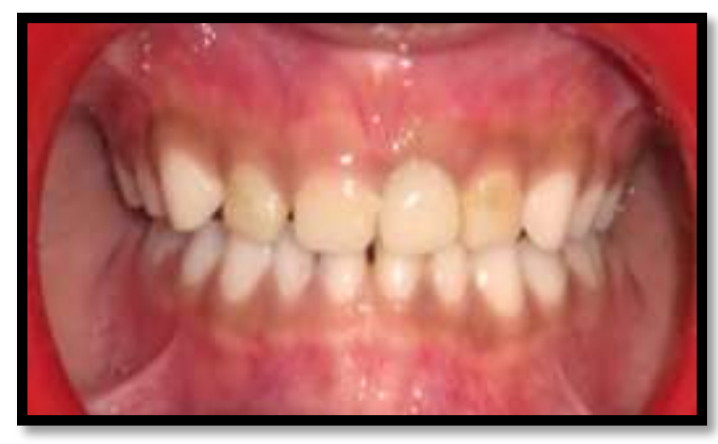

Fig 3:- Post operative photograph of Fixed functional appliance

\section{Case Report 3:-}

A 4 years old female patient reported to the department of Pediatric and Preventive Dentistry, NPDCH with chief complaint of complete knocked out tooth in relation to upper right front tooth region occurred 4 days prior due to trauma. Patient underwent trauma before 4 days and completely knocked out her tooth in relation to upper right front region (51). Patient had pain at the time of accident and gradually relived after sometime.

On clinical examination, 51 was avulsed due to trauma.(Figure 1) And partial edentulous area was seen. Treatment plan for 51 was decided as fixed esthetic functional space maintainer.

Upper and lower alginate impression was made. The mesiodistal width of 61 was measured. A flowable composite in a strip crown was used to fabricate a replacement crown. This was then attached to the adjacent teeth in vivo by etching and bonding the Ribbond with Composite(Figure 2 ). Occlusion was evaluated.

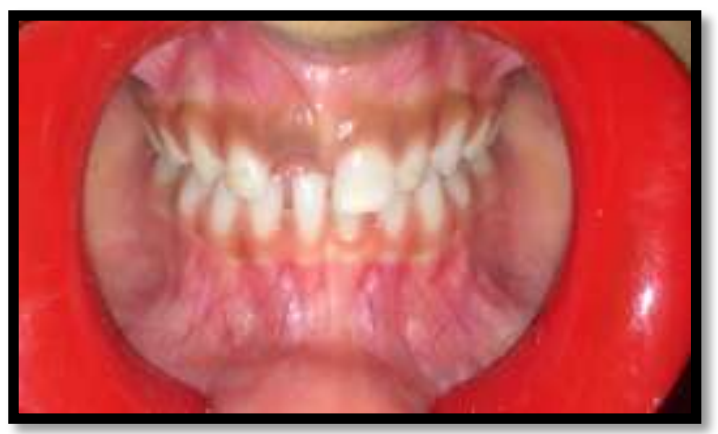

Fig 1:- Preoperative photograph-avulsed 51

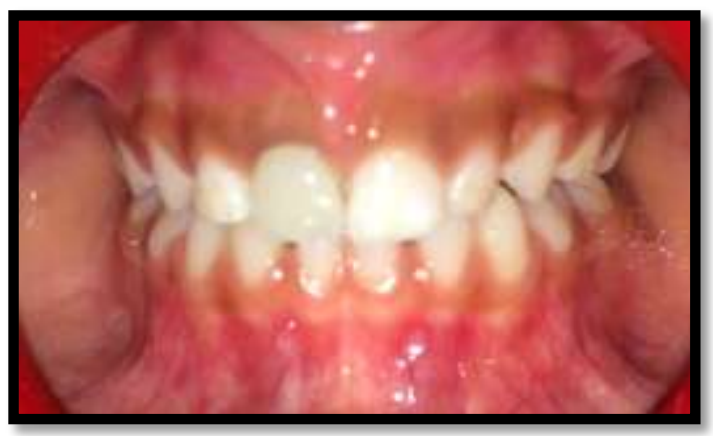

Fig 2:-Post operative photograph of Fixed functional appliance

\section{Discussion:-}

Premature loss of a primary tooth is one of the common causes for malocclusion in permanent dentition. This is mainly because the teeth adjacent to the space created by the loss of the deciduous tooth tend to drift into the space resulting in tooth material arch length discrepancy thereby predisposing the dentition to malocclusion ${ }^{3}$.

In a situation where an anterior tooth is prematurely lost, a removable functional space maintainer is the one, most commonly employed. This requires patient compliance and may have a harmful effect on the involved soft tissue and periodontium. A fixed appliance is usually free from such shortcomings ${ }^{3}$.

Variety of fixed esthetic space maintainers to replace a prematurely lost anterior tooth are in clinical use. The most commonly used technique involves the adaptation of stainless steel bands on the second deciduous molars and a wire soldered to them joining the two bands. The wire is adapted such that it runs close to the palatal surfaces of all teeth anterior to the teeth banded. An additional wire attachment is soldered to this wire which extends over the 
edentulous space and holds the pontic in place. The problem with this appliance is its excessive flexibility and lack of support. In addition, the technique is very complicated and time consuming ${ }^{3}$.

The technique used presently, makes use of Fiber Reinforced Composite resin(FRC) for the fabrication of a fixed esthetic functional space maintainer. FRC is relatively new to Pediatric dentistry, but has been broadly used in removable Prosthodontics, fixed partial dentures, periodontal splints, and in orthodontic treatment as a retention splint $^{5}$. Fiber reinforced composite resin have also been used in the past to replace missing teeth ${ }^{7}$.

In the present case, a freehand build up/strip crowns were utilized, with light cure composite resin to replace the lost primary central incisor. The natural crown of the extracted tooth can also be used. This provides added advantage of preservation of natural tooth form, contour and translucency. Immediate replacement of an extracted tooth using FRC with the natural tooth as the pontic has been successfully tried by a number of investigators ${ }^{\mathbf{8}, \mathbf{1 0}}$.

The techniques utilized here were very simple and the results very esthetic and functionally satisfactory. More clinical research is required with larger number of samples to assess the long-term success of the appliance.

\section{Conclusion:-}

The first-line treatment of non-restorable traumatically injured or carious deciduous teeth is extraction which may hinder the future dentition as well as social activity of a child. Various therapeutic modalities from removable partial dentures to fixed space maintainer can be used for replacement of such lost teeth. Loss of an anterior tooth at younger age may result in psychological trauma to the child. Early loss of a deciduous tooth may also result in drifting of adjacent teeth leading to space loss. Thus, to address the functional and esthetic problem associated with the loss of an anterior tooth, the present appliance was placed using a simple technique with an esthetic and functionally satisfactory result.

\section{References:-}

1. Garai D, Ghosh C, Mandal PK, Kar S. Esthetic anterior fixed functional space maintainer. Int J Pedod Rehabil 2017; 2:90-2.

2. Marwaha M, Bhat M, Nanda KDS. Building-up a Smile in a 5-Year-Old Child: A Case Report. Int J Clin Pediatr Dent 2012;5(2):151-154.

3. Goenka P, Sarawgi A, Marwah N, Gumber P, Dutta S. Simple Fixed Functional Space Maintainer. Int J Clin Pediatr Dent 2014; 7(3):225-228.

4. Ravi.B. Patil, Rachappa.M.M. A Simple Modification Of Fixed Space Maintainers For Replacement Of An Avulsed Maxillary Primary Central Incisor. International Journal Of Dental Clinics January-March 2011; Volume (3) Issue 1.

5. Kargul B, Caglar E, Kabalay U. Glass fiber-reinforced composite resin as fixed space maintainers in children: 12-month clinical follow-up. J Dent Child 2005;72:109-112.

6. Rappelli G, Coccia E. Fiber-reinforced composite fixed partial denture to restore missing posterior teeth: a case report. J Contemp Dent Pract 2005; 4:168-177.

7. Vallittu PK. Case report: a glass fiber reinforced resin bon-ded fixed partial denture. Eur J Prosthodont Restor Dent 2001; 9:35-39.

8. Auplish G, Darbar UR. Immediate anterior tooth replacement using fibre-reinforced composite. Dent Update 2000; 27:267-270.

9. Chauhan M. Natural tooth pontic fixed partial denture using resin composite-reinforced glass fibers. Quintessence Int 2004;35:549-553. 12

10. Ulusoy AT, Cehreli ZC. Provisional use of a natural tooth crown following failure of replantation: a case report. Dent Traumatol 2008; 24:96-99. 